\title{
Vestibulo-Oculomotor Reflex Recording Using the Scleral Search Coil Technique. Review of Peripheral Vestibular Disorders
}

\author{
Marisol Boleas-Aguirre ${ }^{a}$ Amerio A. Migliaccio $^{\mathrm{b}, \mathrm{c}}$, and John P. Carey ${ }^{\mathrm{b}}$ \\ aClínica Universitaria de Navarra, Facultad de Medicina, Universidad de Navarra, Pamplona, \\ Navarra, Spain \\ bDepartamento de Otorrinolaringología y Cirugía de Cabeza y Cuello, Johns Hopkins Hospital, \\ School of Medicine, Johns Hopkins University, Baltimore, United States \\ 'Departamento de Ingeniería Biomédica, Johns Hopkins University, Baltimore, United States
}

\begin{abstract}
Our goal is to review vestibulo-oculomotor reflex (VOR) studies on several peripheral vestibular disorders (Ménière's disease, vestibular neuritis, benign paroxysmal positional vertigo, superior canal dehiscence syndrome, and vestibular neuroma), using the scleral search coil (SSC) technique. Head movements are detected by vestibular receptors and the elicited VOR is responsible for compensatory 3 dimensional eye movements. Therefore, to study the VOR it is necessary to assess the direction and velocity of 3 dimensional head, and eye movements. This can be achieved using the SSC technique. Interaction between a scleral search coil and an alternating magnetic field generates an electrical signal that is proportional to eye position. Ideally, eye rotation axis is aligned with head rotation axis and VOR gain (eye velocity/head velocity) for horizontal and vertical head rotations is almost 1. The VOR gain, however, for torsional head rotations is smaller and about 0.7 .
\end{abstract}

\section{Keywords}

Scleral search coil; Vestibulo-oculomortor reflex; Peripheral vestibular disorders

\section{INTRODUCTION}

The aim of this work is to revise the vestibulo-oculomotor reflex (VOR) recording using the magnetic field scleral search coil (MFSSC) technique for different vestibular diseases:

Ménière's disease, vestibular neuritis, benign paroxysmal positional vertigo (BPPV), superior semicircular canal dehiscence syndrome (SSCDS), and vestibular neurinoma.

\section{VOR 3-DIMENSIONAL RECORDING}

Head movements detected by vestibular receptors trigger the VOR, which generates 3dimensional eye movements.

Correspondence: Dra. M. Boleas-Aguirre., Departamento de Otorrinolaringología., Clínica Universitaria de Navarra., Avda. Pío XII, 36. 31008 Pamplona. Navarra. España., msboleas@unav.es.

The SEORL awarded the first-named author a "Foreign Hospital Visiting Scholarship" for the completion of a Post-doctoral Fellowship at the Department of Otorhinolaryngology and Head and Neck Surgery of the Johns Hopkins Hospital School of Medicine, Johns Hopkins University, in Baltimore, United States. This paper forms part of the studies conducted during that period of training.

The authors have not indicated any conflict of interest. 
In order for the VOR to keep images stable in the retina during head rotations in every direction, it should, ideally, generate compensatory eye movements that meet the following conditions: a) the speed of the eye should be the same as that of the head; $b$ ) the ocular rotation axis should be lined up with the axis of rotation of the head; and $c$ ) the compensatory eye speed should be in synch with the head speed, but in the opposite direction. The lack of coupling in eye speed magnitude or direction triggers a shift in the retinal image during head movement.

In order to study how the VOR works it is necessary to record the direction and speed of horizontal, vertical and torsional head, and eye movements. For that purpose, different 3 dimensional systems have been developed for recording eye and head movements, such as the MFSSC method, and digital recording of eye movements using video cameras. The latter is less aggressive toward the patient than the former, but it is not precise enough in detecting quick eye movements. However, the MFSSC method, although more invasive, is the current standard for recording 3-dimensional eye and head movements because it allows for precise recordings (Figure 1). This articles refers to the basic concepts regarding the bases of the MFSSC method and its usefulness in the study of the VOR.

Three-dimensional eye and head rotations should be recorded within a reference framework consisting of 3 axes: vertical, horizontal, and torsional.

The position of a certain object in space is defined by the angle measurements that make up these 3 reference axes. In this way, eye and head movements can be shown in different ways and, by using different mathematical formulas, 1 can be turned into another. ${ }^{2}$

\section{BASIS AND METHOD OF RECORDING EYE AND HEAD MOVEMENTS BY MFSSC}

Recording eye and head positions by the MFSSC method is based on how the coil interacts with a magnetic field to generate an electric current. The magnetic field is made up of at least 2 pairs of metallic coils placed in a cube measuring one metre per side (Figure 1). These metallic coils create alternate orthogonal magnetic fields. The scleral search coils are made up of 2 orthogonal metallic coils in a single silicone ring (Figure 2). This is placed on the scleral eye surface so that the pupil and iris are left uncovered. The interactions between the scleral coils generate electric signals in them. These signals differ according to the coil position within the magnetic field, which thus reflects the eye's position within the magnetic field. In this way, the position of the eye can be known. A double scleral search coil set on a piece of apparatus that the patient holds in his mouth is also used to measure head speed.

The patient is then seated inside the box where the magnetic fields are. With the help of a laser system, he or she is located in the middle of these. The patient must also be properly placed so that the bony orbital ridge is in the middle of the magnetic fields. The patient's head is placed in such a way that the Frankfurt line is horizontal in relation to the ground. (The Frankfurt line extends from the uppermost area of the external auditory canal's osteocartilaginous union on the ipsilateral infraorbital ridge.)

In spite of wide-ranging head movements, you must make sure that the eye and head coils are located in the linear area of the magnetic field, which roughly corresponds to the volume of a cube with $20 \mathrm{~cm}$ sides. To this end, a device may be used to determine the relative shift of the head in relation to each magnetic field during the head movements so that the head is in the area in which the magnetic fields are linear. Other authors ${ }^{3}$ detail one of the MFSSC recording systems currently used. 


\section{VOR RECORDING WITH MFSSC}

The MFSSC method allows for VOR recording during the head impulse test, which clinically measures the VOR at high frequency stimulations on the horizontal plane. ${ }^{4}$ This manoeuvre consists of a passive head rotation, unpredictable by the patient, of low range $\left(10^{\circ}-20^{\circ}\right)$, high speed $\left(200^{\circ}-400^{\circ} / \mathrm{s}\right)$, and high acceleration $\left(3000^{\circ}-4000^{\circ} / \mathrm{s}^{2}\right)$. Through the MFSSC method the eye rotation axis was studied, the gains (eye/head speed) and VOR latencies triggered by head rotations on the horizontal plane (yaw), the vertical plane (pitch), and frontal plane (roll) (Figure 3). Thus, a check is made as to whether or not the eye and head rotation axes are aligned. The impulse gain on the frontal plane is $0.7(0.08)(95 \%$ CI) (counter clockwise impulse) and 0.74 (0.07) (clockwise); on the vertical plane it is 0.97 (0.05) (upbeating impulse), and 1.09 (0.09) (downbeating), and on the horizontal plane, 0.94 (0.06) (impulse to the right), and 1 (0.07) (to the left). The average latency (standard deviation) of the VOR for impulses on the frontal plane is $10.3(1.9) \mathrm{ms}$; impulses on the vertical plane, $7.6(2.8) \mathrm{ms}$, and for impulses on the horizontal plane, 7.5 (2.9) ms. So, under normal conditions, the VOR generates eye movements that are almost perfectly compensatory regarding head direction and speed movements on the horizontal, and vertical planes. However, for impulses on the frontal plane, even though the eye movements are aligned well, their speed is approximately $30 \%$ slower. $^{1}$ This agrees with what other authors have found. $5^{-7}$

The MFSSC method may also be used to record the VOR in response to head rotations on the planes of the pairs of collinear semicircular canals: $a$ ) on the horizontal plane for the stimulation of horizontal semicircular canals; $b$ ) on the RALP (right anterior, left posterior) plane for right anterior and left posterior semicircular canals and $c$ ) on the LARP (left anterior, right posterior) plane for activating the left anterior and right posterior semicircular canals. To trigger stimuli on the RALP and LARP planes, the researcher puts 1 hand in front on the forehead and the other on the patient's occipitus so that the head rotation is aligned with each of these planes, respectively. Then short and abrupt impulses are done with one of the hands while the other is used as a guide (the impulses have been described previously). It is important to do the head impulses on the planes in this way so that, on the 1 hand, there is maximum stimulation of each pair of semicircular canals and, on the other hand, horizontal rotation is avoided. This shows that, in patients who do not suffer from vestibular problems, the average VOR (eye/head speed) gain, with a 95\% CI in horizontal impulses at maximum head speed, is $0.9(0.1)$. However, in diagonal head impulses (RALP and LARP), the VOR gain is approximately $0.7-0.8$. When the eye and head speeds are broken down into their rotational components in the vertical and torsional planes, we can see that the eye rotational component on the vertical plane is around 0.9 and the gain of the eye rotational component on the frontal plane is approximately 0.6.

Therefore it seems that the lack in gain of the diagonal VOR (RALP and LARP planes) is due to a lack in gain of the frontal plane rotational component. ${ }^{8}$ This agrees with previous comments.

\section{MÉNIÈRE'S DISEASE}

In patients suffering from Ménière's disease, results from the caloric test (which explore the low-frequency stimulation range between $0.01-0.03 \mathrm{~Hz}$ ) were compared with the angular VOR gain from using the MFSSC method during the eye-head tracking (recording the response to high frequency stimuli).

In patients in the active phase of the disease who have frequent vertigo attacks, a pathological caloric test was observed in most of them, while the eye-head manoeuvre is impaired in fewer cases. Therefore, Ménière's disease itself seems to cause a slight decrease in the VOR gain during the eye-head manoeuvre. It has been stated that this is due to the fact that these patients still conserve vestibular function, especially at high frequencies. ${ }^{9}$ 
In the same way, it is also seen that the horizontal VOR, recorded by the MFSSC method, seems to be in correlation with vertigo control following intratympanic gentamycin treatment. However, there is no link between vertigo control and post-therapeutic results of the caloric test. Also, by using the MFSSC method, it can be seen that those patients who have good vertigo control with an intratympanic gentamycin shot, show higher levels of impairment of the ipsilateral horizontal VOR symmetry and gain than those patients who need more injections to control their vertigo. It was noted that a $50 \%$ decrease in the horizontal VOR gain after intratympanic gentamycin treatment is linked to good vertigo control. ${ }^{10}$

On another note, it was sometimes observed that patients with Ménière's disease who underwent vestibular nerve neurectomy, with hearing kept intact, may suffer from vertigo attacks. For these cases, by using the MFSSC method during the eye-head manoeuvre, it was observed that residual function in the posterior semicircular canal was still present, probably due to an incomplete neurectomy. ${ }^{11}$

\section{VESTIBULAR NEURITIS}

Vestibular neuritis is a syndrome derived from a sudden and complete loss of peripheral vestibular function characterized by vertigo, nystagmus, dizziness, nausea, and vomiting. The nystagmus that appears is mostly horizontal, having a torsional component and whips back to the opposite side of the lesion. It is due to a condition (viral, vascular, etc) of the vestibular nerve. At least in the initial stages of vestibular neuritis, there is caloric hypofunction, which means that the horizontal semicircular canal and its pathway are diminished. ${ }^{12}$ Normally the superior vestibular nerve conducts efferences from the superior semicircular canal, horizontal semicircular canal, utricle, and part of the saccule, while the inferior vestibular nerve conducts the efferences from the posterior semicircular canal and most of the saccule. In those patients with vestibular neuritis it seems that the superior vestibular nerve is the one that is affected most.13

By using the MFSSC method for studying eye movement in vestibular neuritis cases, it is noted that the spontaneous nystagmus axis aligns with the horizontal semicircular canal or that resulting from the horizontal and superior semicircular canals. There is also VOR asymmetry and gain decrease. 14, 15 These findings indicate that only the superior division of the vestibular nerve is affected. This is also confirmed because the patients with neuritis may show PSC BPPV (which implies intact posterior semicircular canal function) and they have vestibular myogenic evoked potentials (which means that the saccule function and its pathway are intact). Therefore in these cases the inferior vestibular nerve is not affected. ${ }^{13}$ By using the MFSSC method in the study of patients with vestibular neuritis, 3 different patterns of impairment of semicircular canal functions have been observed: $a$ ) alteration of just the horizontal function, or a combination of the horizontal and superior functions; $b$ ) loss of the horizontal, superior, and posterior functions; and $c$ ) alteration of just the posterior function. Taking into account the innvervation distribution in the superior and inferior vestibular nerve divisions, the alteration of just the horizontal semicircular canal function, or the combined horizontal and superior ones, means that there is selective impairment of the superior vestibular branch. If the function of the 3 canals is altered, the patient will have vestibular neuritis affecting the entire vestibular nerve. On the other hand, if only the posterior semicircular canal function is found to be pathological, then only the inferior vestibular nerve is affected. By using the MFSSC methods these authors have found isolated hypofunction of the posterior semicircular canal in patients with clinical vestibular neuritis symptoms, but with normal caloric test and horizontal eye-head tracking, as well as associated hypoacusia. The lack of clinical diagnosis of inferior vestibular nerve neuritis is probably due to the fact that these patients are not diagnosed with vestibular neuritis, but central vestibular syndromes instead. This may have happened because the 
spontaneous nystagmus that appears is not intense, nor horizontal, but downbeating vertical and torsional, and it also has a normal caloric test as well. ${ }^{12,13}$

\section{BENIGN PAROXYSMAL POSITIONAL VERTIGO}

The diagnosis of BPPV is based on recognizing, during clinical examination, the typical characteristics of the positional nystagmus that goes along with it. Most frequently a mixed vertical and geotropic upbeating nystagmus due to posterior semicircular canal canalithiasis is seen. A horizontal geotropic nystagmus due to horizontal semicircular canal canalithiasis or a horizontal ageotropic nystagmus due to horizontal semicircular canal cupulolithiasis may also be seen. We rarely see vertical downbeating nystagmus with a geotropic-torsional component due to a superior canalithiasis.

However, when the positional nystagmus is atypical or the BPPV is treatment resistant, diagnosis and cure are more complicated. In these types of patients, Aw et a ${ }^{16}$ have conducted 3-dimensional recording and vector analysis of eye movements using the MFSSC method. They put the patients in a system that allows for rotation on 2 axes and performed Dix-Hallpike and Lempert manoeuvres simultaneously with eye movement recordings. In this way, they studied the positional nystagmus vector speed and position. They compared it with each semicircular canal's axis in order to determine which were affected, and then did the appropriate repositioning manoeuvre. The rotation axis of the vestibular nystagmus is orthogonal to the plane of the stimulated semicircular canal. This derives from Edwald's first law, from observing eye movements induced by electrical stimulation of the semicircular canal nerves, 17 from the findings of the superior semicircular canal dehiscence syndrome, 18 and from studies done on BPPV.19-22 It has been seen that, in bilateral posterior semicircular canal BPPV, the magnitude of the positional nystagmus is similar in stimulation on both sides, the vertical component is always upbeating, but the torsional one changes direction in each case, and it becomes geotropic-torsional (toward the lowermost ear). In cases of canalithiasis and cupulolithiasis of the horizontal semicircular canal, it is noted that there is a torsional component besides the geotropic and ageotropic horizontal ones, respectively. This torsional component of the nystagmus from horizontal semicircular canal stimulation appears because this canal is located $30^{\circ}$ higher with respect to the horizontal one. Nystagmus duration is also longer in cupulolithiasis than in canalithiasis. The presence of a downbeating positional nystagmus in the Dix-Hallpike manoeuvre is attributed to superior semicircular canal BPPV in patients who do not have a central alteration. These authors confirm that the Dix-Hallpike manoeuvre toward either side triggers superior semicircular canal positional nystagmus with a small associated torsional component. The cases are resolved by using a repositioning manoeuvre that consists of a forward rotation on the affected superior semicircular canal plane. That study showed the usefulness of the MFSSC method in recording patients with mixed positional vertigo, who are not easily diagnosed through a physical exam. It specifically presents patients whose eye rotational axis in positional nystagmus has an oblique orientation and a disproportionately large geotropic-torsional component in the Dix-Hallpike manoeuvre as well as the Lempert one. For these cases, the result of eye movement analysis by the MFSSC method shows that the eye rotation vector is located between the axis that corresponds to the stimulation of the posterior semicircular canal and that of the horizontal semicircular canal, indicating a mixed posterior and horizontal BPPV. ${ }^{16}$

On the other hand, in those BPPV patients who underwent occlusion of the corresponding semicircular canal, an abrupt and permanent decrease of the VOR in the eye-head manoeuvre was seen when using the MFSSC method in the direction that the occluded semicircular conduct would stimulate. ${ }^{7}$ 


\section{SUPERIOR SEMICIRCULAR CANAL DEHISCENCE SYNDROME}

Superior semicircular canal dehiscence syndrome (SSCDS), as its name indicates, appears as a consequence of temporal bone dehiscence on the superior semicircular canal. The typical symptoms are vertigo and oscillopsia induced by intense noises, or changes in the intracranial pressure or middle ear. Other things that may occur are transmission hypoacusia and an increase in the bone-conducted auditory threshold. These manifestations are seen because the dehiscence creates a third mobile window in the inner ear. Diagnosis is effected by clinical presentation of the vertical-rotational nystagmus triggered by sound or pressure, which happens when the corresponding superior semicircular canal is stimulated, a decrease in the threshold and an increase in the range of the vestibular myogenic evoked potentials and dehiscence images of the superior semicircular canal in high-resolution computerized tomography of the petrous portion of the bone $(0.5 \mathrm{~mm}$ slices and reconstruction in the plane of the canal).

In SSCDS, it can be seen that exposures to intense noises, positive pressure in the outer ear canal and/or applying the Valsalva manoeuvre with a plugged nose (open glottis) trigger an ampullofugal (excitatory) deflection of the superior semicircular canal cupola. This triggers a conjugate eye movement whose slow phase has an upbeating vertical component and a torsional component in which the superior eye pole drifts toward the unaffected side. However, by applying negative pressure in the external ear canal, Valsalva's manoeuvre with a closed glottis and jugular pressure generate an ampullopetal (inhibitory) deflection in the superior semicircular canal cupola, evoking a downbeating vertical eye movement with a torsional component toward the affected ear. ${ }^{23}$ The typical eye movements triggered can be studied by using the MFSSC method. It has been shown, then, that the eye movements are aligned with the plane of the affected canal.18

\section{VESTIBULAR NEURINOMA}

Patients with vestibular neurinomas may have hyperventilation-induced nystagmus. Studying this nystagmus by using the MFSSC method shows that in its slow phase the affected vestibular nerve is stimulated. It seems that hyperventilation triggers a temporary increase in the partially demyelinated axons as a result of the neurinoma. Eye rotational axis during nystagmus shows that the horizontal semicircular canal nerve fibres are the ones that are activated the most. The superior semicircular canal ones are activated to a lesser degree and the posterior semicircular canal ones have varying degrees of activation. ${ }^{24}$

\section{REFERENCES}

1. Aw ST, Halswanter T, Halmagyi GM, Curthoys IS, Yavor RA, Todd MJ. Three-dimensional vector analisis of the human vestibuloocular reflex in response to high-acceleration head rotations. I. Responses in normal subjects. J Neurophysiol 1996;76:4009-4020. [PubMed: 8985896]

2. Halswanter T. Mathematics of three-dimensional eye rotations. Vision Res 1995;35:1727-1739. [PubMed: 7660581]

3. Migliaccio A, Della Santina C, Carey J, Minor L, Zee D. The effect of binocular eye position and head rotation plane on the human torsional vestibuloocular reflex. Vision Res 2006;46:2475-2486. [PubMed: 16545855]

4. Halmagyi GM, Curthoys IS. Aclinical sign of canal paresis. Arch Neurol 1988;45:737-739. [PubMed: 3390028]

5. Collewijn H, Van der Steen J, Ferman L, Jansen TC. Human ocular counter-roll: assessment of static and dynamic properties from electromagnetic scleral coil recordings. Exp Brain Res 1985;59:185196. [PubMed: 4018196]

6. Crawford JD, Vilis T. Axes of eye rotation and Listing's Law. J Neurophysiol 1991;65:407-423. [PubMed: 2051188] 
7. Leigh RJ, Maas EF, Grossman GE, Robinson DA. Visual cancellation of the torsional vestibulo-ocular reflex in humans. Exp Brain Res 1989;75:221-226. [PubMed: 2721607]

8. Cremer PD, Halmagyi GM, Aw ST, et al. Semicircular canal plane head impulses detect absent function of individual semicircular canals. Brain 1998;112:699-716. [PubMed: 9577395]

9. Park HJ, Migliaccio AA, Della Santina CC, Minor LB, Carey JP. Search-coil head-thrust and caloric tests in Meniere's disease. Acta Otolaryngol 2005;125:852-857. [PubMed: 16158532]

10. Lin FR, Migliaccio AA, Haslwanter T, Minor LB, Carey JP. Angular vestibule-ocular reflex gains correlate with vertigo control after intratympanic gentamicin treatment for Meniere's disease. Ann Otol Rhinol Laryngol 2005;114:777-785. [PubMed: 16285268]

11. Lehnen N, Aw ST, Todd MJ, Halmagyi GM. Head impulse test reveals residual semicircular canal function after vestibular neurectomy. Neurology 2004;62:2294-2296. [PubMed: 15210899]

12. Aw ST, Fetter M, Cremer PD, Karlberg M, Halmagyi GM. Individual semicircular funtion in superior and inferior vestibular neuritis. Neurology 2001;57:768-774. [PubMed: 11552001]

13. Halmagy GM, Aw ST, Karlberg M, Curthoys IS, Todd MJ. Inferior vestibular neuritis. Ann N Y Acad Sci 2002;956:306-313. [PubMed: 11960814]

14. Fetter M, Dichgans J. Vestibular neuritis spares the inferior division of the vestibular nerve. Brain 1996;119:755-763. [PubMed: 8673488]

15. Schmid-Priscoveanu A, Bohmer A, Bocina H, Straumann D. Caloric and search-coil head-impulse testing in patients after vestibular neuritis. J Assoc Res Otolaryngol 2001;2:72-78. [PubMed: 11545152]

16. Aw ST, Todd MJ, Aw GE, McGarvie LA, Halmagyi GM. Benign positional nystagmus. Astudy of its 3-dimensional spatio-temporal characteristics. Neurology 2005;64:1897-1905. [PubMed: 15955941]

17. Cohen B, Suzuki JI, Bender MB. Eye movements from semicircular canal nerve stimulation in the cat. Ann Otol Rhinol Laryngol 1964;73:153-169. [PubMed: 14128701]

18. Cremer PD, Minor LB, Carey JP, Della Santina CC. Eye movements in patients with superior semicircular canal dehiscence aligns with the abnormal canal. Neurology 2000;55:1833-1841. [PubMed: 11134382]

19. Fetter M, Sievering F. Three-dimensional eye movement analysis in benign paroxysmal positioning vertigo and nystagmus. Acta Otolaryngol 1995;115:353-357. [PubMed: 7653253]

20. Imai T, Takeda N, Uno A, Morita M, Koizuka I, Kubo T. Three dimensional eye rotation axis analysis of benign paroxysmal positioning nystagmus. ORL J Oto-rhino-laryngol Relat Spec 2002;64:417423.

21. Hayashi Y, Kanzaki J, Etoh N, et al. Three-dimensional analysis of nystagmus in benign paroxysmal positional vertigo. New insights into its pathophysiology. J Neurol 2002;249:1683-1688. [PubMed: 12529790]

22. Imai T, Takeda N, Ito M, et al. Three-dimensiontal analysis of benign paroxysmal positional nystagmus in a patient with anterior semicircular canal variant. Otol Neurotol 2006;27:362-366. [PubMed: 16639275]

23. Minor LB. Clinical manifestations of superior semicircular canal dehiscence. Laryngoscope 2005;115:1717-1727. [PubMed: 16222184]

24. Minor LB, Haslwanter T, Straumann D, Zee DS. Hyperventilation-induced nystagmus in patients with vestibular schwannoma. Neurology 1999;53:2158-2168. [PubMed: 10599798] 


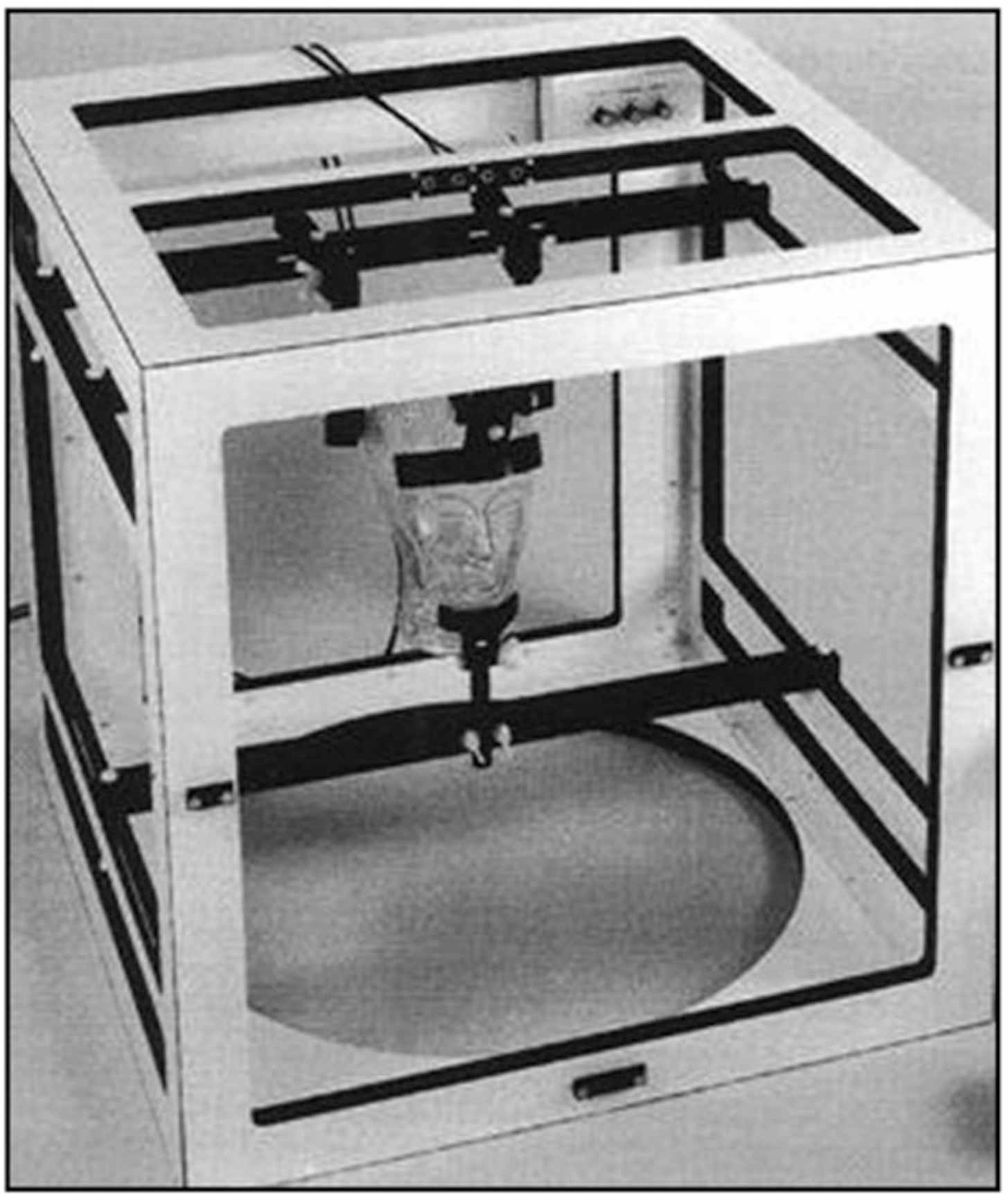

Figure 1.

Structure on which the magnetic field generating system is mounted. 

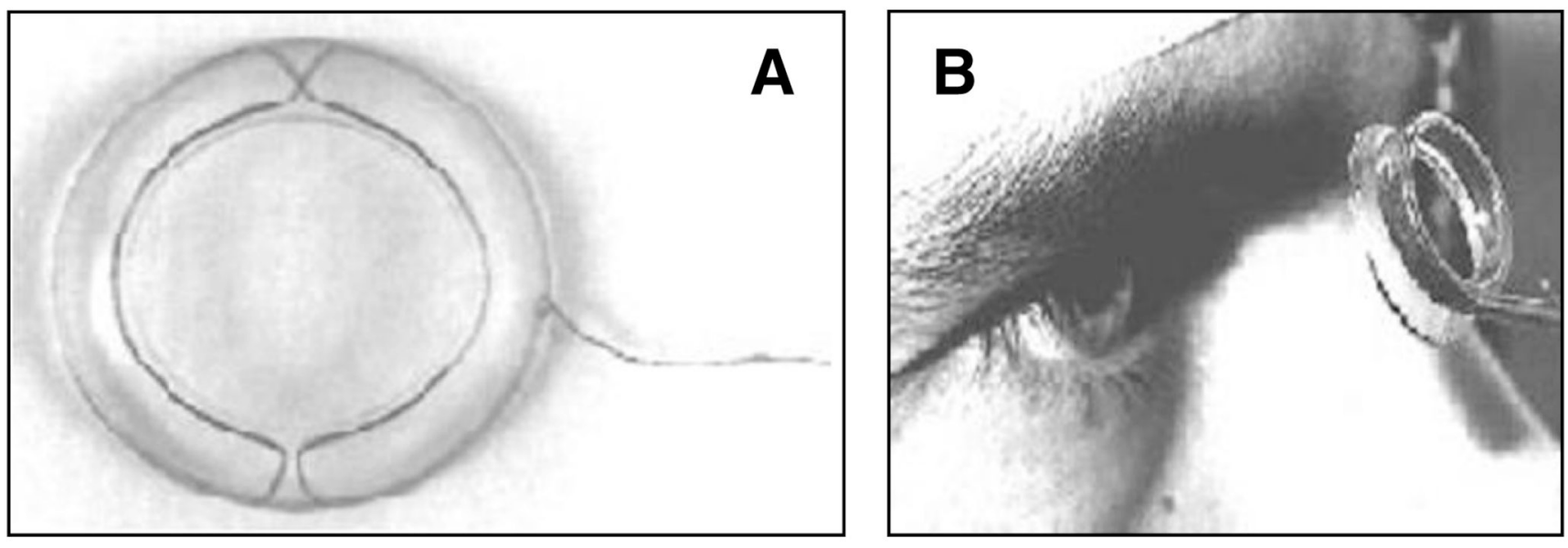

Figure 2.

Scleral coils (A) and placement on the patient (B). 


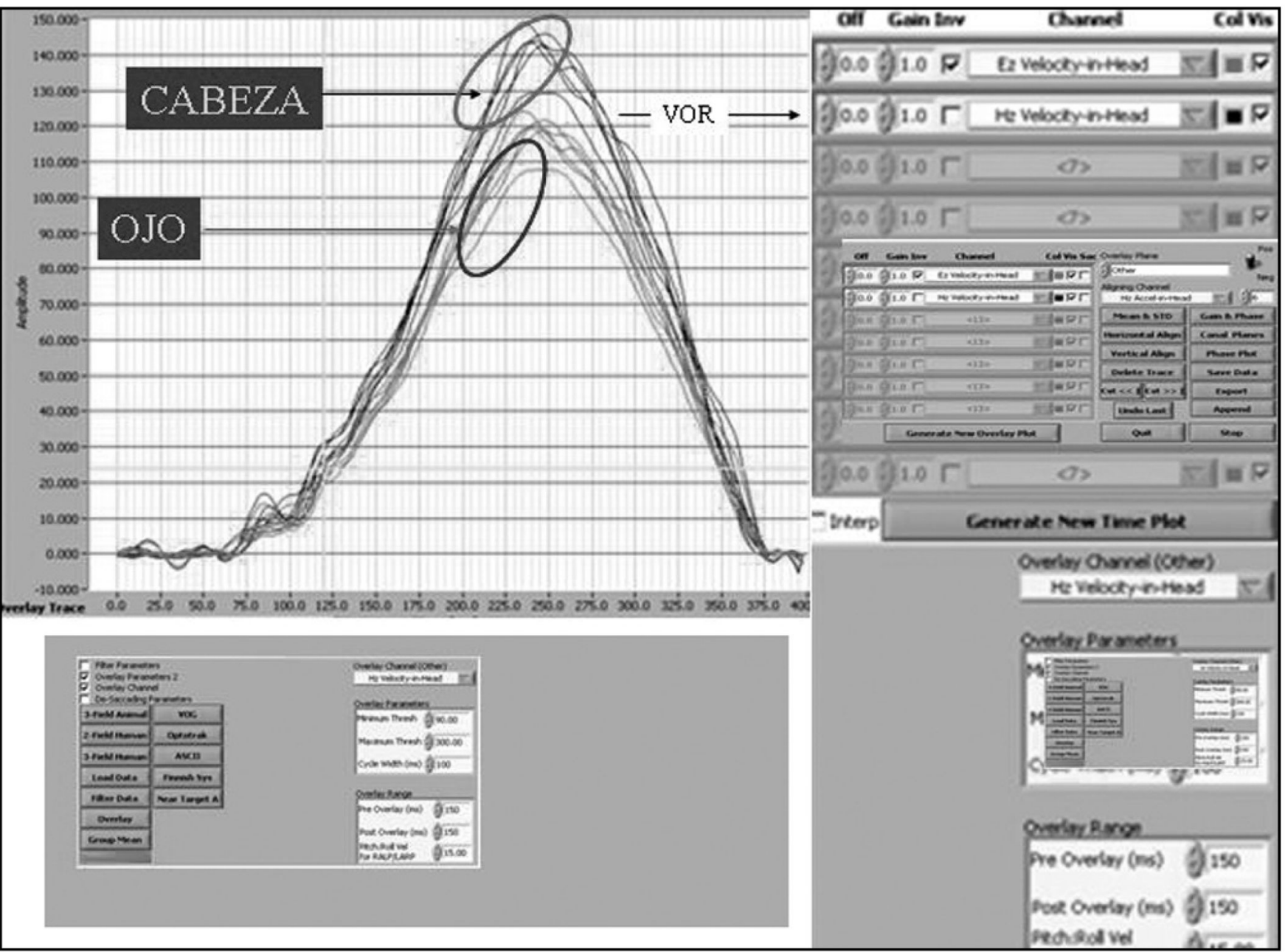

Figure 3.

Example of an eye and head movement recording using the scleral search coil technique in a magnetic field. 